\title{
Transactive discourse in CSCL
}

\author{
Gerry Stahl
}

Published online: 25 April 2013

(C) International Society of the Learning Sciences, Inc. and Springer Science+Business Media New York 2013

The previous two issues of $i j C S C L$ explored the multiple levels of analysis characteristic of CSCL research. In this issue, we look at multiple levels in four more papers. Two of them explicitly discuss the notion of 'transactivity' in this context. They all consider how students - from kindergarten to college — build on each other's reasoning through the shared use of computer media - synchronously or asynchronously - and how this can be measured and supported.

The concept of transactivity connotes a spanning of activity across multiple actors. Defining transactivity as the reasoning of one utterance building on another utterance's reasoning suggests two ways of looking at transactions: in terms of the distinct utterances of the individuals or the unified interaction in the dyad, small group or community.

The first approach reduces the description to the individual unit of analysis. An individual A expresses his or her individual cognition (mental model, internal representation, thought, reasoning) in an utterance $\mathrm{P}$, and then individual $\mathrm{B}$ observes utterance $\mathrm{P}$, interprets $\mathrm{P}$ in terms of B's mental model of A's intentions and formulates a transactive utterance $\mathrm{Q}$ in response. The sequence P-Q may build knowledge or express a logical argument. The sequence would not have occurred through the mental activity of $\mathrm{A}$ or $\mathrm{B}$ alone, but results from the interaction of $\mathrm{A}$ and $\mathrm{B}$, in which $\mathrm{B}$ builds on $\mathrm{A}$. Yet, the entire transaction has been analyzed in terms of mental states of the individuals A and B. One can, for instance, go on to ask about the extent to which $\mathrm{A}$ and $\mathrm{B}$ had similar individual understandings of $\mathrm{P}$ and $\mathrm{Q}$.

Alternatively, it is possible to analyze the transaction P-Q at the group unit of analysis. One can say, we know nothing about internal states of A and B other than what is implicit in what they say aloud in order to make their meanings clear to each other. As observers or analysts, we can understand what they say in the same ways that they understand each other - on the basis of how they make themselves clear to each other-assuming that we have a similar cultural background and understand their language. In this approach, the meaning of the transaction is located in the pair of utterances, $\mathrm{P}-\mathrm{Q}$, rather than in the minds of A or B. P may be understood in terms of its elicitation of a response such as Q; P opens the possibility of such a response and this possibility is an essential aspect of its meaning. $Q$

G. Stahl $(\bowtie)$

Drexel University, Philadelphia, PA, USA

e-mail: Gerry@ijCSCL.org 
responds to P; the meaning of Q is highly dependent upon P. It may be better to say that the meaning does not lie partially in P taken on its own and partially in Q by itself, but in the unity P-Q. The meaning may involve semantic, syntactic and pragmatic references between $\mathrm{P}$ and $\mathrm{Q}$, which only make sense when $\mathrm{P}$ and $\mathrm{Q}$ are taken together. An analyst of transaction P-Q must understand the meaning of the pair of utterances taken together, situated in their on-going discourse. Such an analysis is at the group level of analysis of the discourse between people. It consists of linguistic analysis of the transactive utterances, not of the mental states of the individual speakers.

As CSCL researchers, we understand the meanings of transactions between subjects in our data because we are members of the same broad community as our subjects. We can give "thick descriptions" of their utterances in terms of what they mean. The term "thick description" comes from the philosopher Austin and the anthropologist Geertz (see the following papers for references). Austin distinguished a thin description of someone's eye twitching vs. a thick description of someone winking. An objective description of the physiology or movement of an eyelid might be the same for a twitch or a wink. However, a wink is part of a meaningful transaction between people and must be understood and described as such. This requires subtle cultural knowledge, which is why Geertz was concerned with how one makes reliable thick descriptions in anthropological contexts involving exotic cultures.

A transactive pair of utterances can serve as a boundary object between CSCL analyses at the individual and small-group unit of analysis. Not only can we relate the different analyses of a specific pair of utterances, but we can also extend the analysis of the transactive utterances to conceptions of individual or group background knowledge, common ground and transactive memory systems.

It is important to note that the original conception of transactivity by Wegner and others in the 1980s was significantly different from more recent theories of distributed and group cognition. It was a psychological theory focused on situations in which individuals hold different knowledge and members of the group engage in transactions to assist in recall of the stored information, largely through meta-knowledge about what each other knows. Thus, transactivity analyzed group phenomena at the individual unit, as contrasted to theories of intersubjective shared understanding, where knowledge is spread across multiple people and their artifacts or where a group is engaged in building and maintaining a joint problem space, which is co-experienced.

The articles in this issue address many of these matters of building knowledge together, each in a quite different way. They each grapple with the issue of units of analysis. They also discuss the ways in which computers can support collaborative knowledge building despite the fact that computers cannot formulate or understand thick descriptions of discourse moves. What is interesting about this collection of CSCL research reports is not so much their commonality in considering levels of analysis, as the rich diversity of their approaches to doing so.

In the first paper, Richard Alterman and Johann Ari Larusson undertake an extended theoretical and empirical analysis of knowledge creation in loosely coordinated learning activities - specifically in student blogging - in contrast to meaning making in a tightly coupled joint problem space, as is more commonly analyzed in CSCL research. While blogging is a predominantly individual writing and reading experience, it also provides for transactive building on the reasoning of other students and, over time, in the emergence of common knowledge from participation in the persistent and growing community blogosphere.

The next article raises the question of how to support transactivity with scripting. Omid Noroozi, Stephanie D. Teasley, Harm J. A. Biemans, Armin Weinberger and Martin Mulder report on a laboratory experiment in which interactions of dyads with different expertise are scripted in ways hypothesized to increase transactivity. One script prompts for building 
awareness about a learning partner's expertise, assigning and accepting task responsibility, and forming a collaboratively shared system for retrieving information based on the partner's specialized expertise. The other script prompts for analyzing arguments put forward by learning partners and constructing arguments that relate to already externalized arguments. The study examines the individual and combined effects of these two kinds of scripts on the quality of both joint and individual problem solutions. Interestingly, each of the scripts - designed to support one aspect of transactivity - seems to be beneficial, but when combined the scripts apparently get in each other's way.

Florencia Gómez, Miguel Nussbaum, Juan F. Weitz, Ximena Lopez, Javiera Mena and Alex Torres explore a situation in which triads of kindergarten children work together on a single computer with three mice and three distinct areas of the screen. In keeping with the theory of transactivity, the participants have different but interdependent roles or tasks. The authors argue that interacting on a single computer increases shared attention, and hence the tendency to build transactively. The children engage in group processes in order to achieve a common goal on the shared screen. Thereby, they develop social skills. Experimental results show that this combination of individual roles and group interaction-typical of transactivity - are effective in enhancing the social skills of the young children, as measured individually by standardized psychological tests. Given the inexperience of the kindergarteners, a teacher must mediate, analogously to the scripted prompts for the college students in the previous study.

The final paper, by Gahgene Gweon, Mahaveer Jain, John McDonough, Bhiksha Raj and Carolyn Rosé, addresses the task of automatically assessing the level of transactivity in a spoken-discourse corpus. Perhaps because computers cannot engage in thick descriptions of the meaning of interactions, an indirect approach is taken here. Data-mining algorithms have become proficient at identifying patterns in thin descriptions of data, such as acoustical features of speech. The authors argue that insights from the social psychology and sociolinguistics of speech style imply that a measure of speech-style accommodation should positively correlate with a prevalence of other-oriented transactions in conversation. That is, people tend to accommodate certain acoustic characteristics of their speech-such as variation and average levels of pitch, intensity of speech or the amount of silence and duration of speech - to that of their partner to roughly the same extent that they engage in building on their partner's reasoning. Thus, an analysis of the acoustics of speech can predict a level of transactive reasoning. 